\title{
Is mold sensitization associated with severe asthma exacerbation in children?
}

\author{
Isree Leelayuwattanakul, Kittipos Visitsunthorn, Witchaya Srisuwatchari, Punchama Pacharn, \\ Orathai Jirapongsananuruk, Nualanong Visitsunthorn
}

\begin{abstract}
Background: Mold sensitization has been reported as a factor associated with severe asthma exacerbation (SAE).

Objective: To identify the factors associated with SAE in asthmatic children, particularly mold sensitization.

Methods: The asthmatic children recruited into this case-control study were classified into an SAE and an outpatient (OPD) group, based on their histories of asthma exacerbation with hospitalization in the preceding year. A skin prick test to common aeroallergens was performed. Possible SAE risk factors were analyzed.
\end{abstract}

Results: A total of 102 patients were enrolled. The 51 patients in the SAE group were significantly younger than the 51 in the OPD group (mean ages of $6.8 \pm 3.3$ vs $8.7 \pm 3.2$ years, $p=0.005$ ). Higher proportions of patients with partly controlled or uncontrolled asthma were found in the SAE group (41.2\% vs $17.6 \%, p=0.009)$. The incidences of a paternal history of atopy, an emergency department visit, and a history of systemic corticosteroid administration in the preceding year were significantly higher for the SAE group $(35.3 \%$ vs $15.7 \%, p=0.023 ; 100 \%$ vs $43.5 \%, p<0.001$; and $100 \%$ vs $31.4 \%, p<0.001$; respectively). The multivariate logistic regression analysis showed that risk factors for SAE were Alternaria sensitization (adjusted odds ratio [AOR] 3.00; 95\% CI 1.09-8.3; $p=0.033$ ), patients who were younger than 6 years (AOR 3.28; 95\% CI 1.17-9.18; $p=0.024$ ), and a paternal history of atopy (AOR 2.94; 95\% CI 1.05-8.25; $p=0.040$ ).

Conclusions: Alternaria sensitization, an age younger than 6 years, and a paternal history of atopy were associated with $\mathrm{SAE}$ in asthmatic children.

Key words: Alternaria, asthma exacerbation, children, mold sensitization, severe asthma

\section{From:}

Division of Allergy and Clinical Immunology, Department of Pediatrics, Faculty of Medicine, Siriraj Hospital, Mahidol University, Bangkok, 10700, Thailand

\section{Introduction}

Asthma is the most common chronic respiratory disease in children. In 2001, the International Study of Asthma and Allergies (ISAAC) Phase III reported that asthma affected $15 \%$ of children in Bangkok. ${ }^{1}$ The most recent study in Thailand in 2019, the Global Asthma Network (GAN) phase I, showed that prevalence of current wheezing in children was $13.5 \% .^{2}$ Asthma exacerbation remains a major health problem for patients of any age. It also affects patients' families and their community. In 2016, nearly $54 \%$ of children with asthma in the USA were reported to have had at least 1 asthma attack in the preceding year, with $4.7 \%$ being hospitalized

\section{Corresponding author:}

Nualanong Visitsunthorn

Division of Allergy and Immunology, Department of Pediatrics,

Faculty of Medicine, Siriraj Hospital, Mahidol University,

2 Wanglang Rd, Bangkoknoi, Bangkok 10700, Thailand

E-mail: nualanongv@yahoo.com

and $16.7 \%$ involving emergency department (ED) visits. ${ }^{2}$ Similarly, a survey of poorly controlled asthmatic children in 12 geographic areas of Asia-Pacific showed that $43 \%$ had had at least one asthmatic exacerbation, $17 \%$ had needed hospitalization, and $19 \%$ had had an ED visit. ${ }^{3}$

Severe asthma exacerbation (SAE) is defined as a hospitalization or ED visit because of worsening asthma, or the need for systemic corticosteroids to control asthma. ${ }^{4}$ SAEs are one of the most common causes of critical illness in children, accounting for approximately 10,000 intensive care unit (ICU) admissions per year in the USA. Children admitted to 
the ICU are significantly more likely to have an allergy or irritant-triggered exacerbation than those admitted to a general ward (OR 3.9; 95\% CI 1.9-8.2; $p=0.0003$ ). ${ }^{5}$ Most children hospitalized for asthma exacerbations are sensitized to multiple indoor allergens. More than $50 \%$ are sensitized to any of Alternaria, Aspergillus, dust mite, cat dander, and dog dander. ${ }^{6}$

Our previous study showed a significant increase in Alternaria sensitization had occurred after the major flooding in Bangkok in 2010.7 Mold, especially Alternaria sensitization, has been reported as a factor associated with SAE. ${ }^{8,9}$ Other reported factors are obesity, second-hand smoke, viral infections (particularly rhinovirus), and poor adherence. ${ }^{10,11,12}$ Elucidating the roles of these factors in SAE may assist with SAE prevention.

The aim of this study was to identify the factors associated with SAE requiring the hospitalization of Thai children, particularly mold sensitization.

\section{Materials and methods \\ Participant recruitment}

The children in this case-control study were recruited from the Pediatric Allergy Clinic, Faculty of Medicine, Siriraj Hospital. Aged 1-15 years and clinically diagnosed as having asthma, their level of asthma control was defined according to the Global Initiative for Asthma criteria. The study was conducted from 2016 to 2018. Its protocol was approved by the Siriraj Institutional Review Board and registered with ClinicalTrials.gov (NCT03690349). Written informed consent and/ or assent was obtained from patients and/or guardians prior to inclusion.

Excluded were patients who were pregnant, lactating, or had a chronic illness (such as heart disease, genetic diseases, and chronic pulmonary diseases). Patients were classified into 2 groups: a severe asthma exacerbation (SAE) group, and an outpatient department (OPD) group. The SAE group was defined as patients who had been hospitalized due to severe asthma exacerbation in the preceding year, whereas the OPD group was defined as patients who had not been hospitalized due to severe asthma exacerbation in a preceding year. The flow of the participants in the study is illustrated in Figure 1.
The following patient data were evaluated: age, sex, body weight, height, family history of atopy, parental income and education, severity of asthma, level of asthma control, asthma medication, adherence to the treatment, comorbidities, environment exposure, and past history of asthma treatment.

Skin prick tests (SPT) were evaluated for 16 common aeroallergen extracts (ALK-Abelló A/S, Hørsholm, Denmark). They included Dermatophagoides pteronyssinus (Dp), Dermatophagoides farinae (Df), American cockroach, German cockroach, cat dander, dog epithelia, mouse epithelium, Bermuda grass, Johnson grass, careless weeds, acacia, Alternaria spp., Cladosporium spp., Penicillium spp., Aspergillus spp. and Curvularia spp.

SPTs were performed with metal lancets by a trained health professional. Histamine dihydrochloride $(10 \mathrm{mg} / \mathrm{mL})$ and normal saline solution were used as positive and negative controls, respectively. The SPT results were recorded 10 and 15 minutes after pricking with histamine and the allergens, respectively. The SPT result was defined as positive if the mean wheal diameter (sum of the widest wheal diameter and the perpendicular diameter, divided by two) was at least $3 \mathrm{~mm}$ larger than that of the negative control.

\section{Statistical analysis}

The sample size and power were calculated based on data reported by a previous study on adult asthma. ${ }^{13}$ We determined that a case-control study with $50 \mathrm{SAE}$ cases and 50 OPD cases would have sufficient statistical power to detect significant $(p<0.05)$ differences in at least one mold sensitization in the SAE group.

The demographic data were summarized using descriptive statistics. The categorical data are presented as number and percentage, and the continuous data are presented as either mean \pm standard deviation or median and range. The factors associated with SAE were analyzed using the chi-square test, Fisher's exact test, and unpaired t-test. Multiple logistic regression analysis was used to determine the effect of each independent factor after controlling for the confounding influence of the other factors. The odds ratios and $95 \%$ confidence intervals (CI) for the two groups were calculated. A $p$-value of less than 0.05 was regarded as being statistically significant. All statistical analyses were performed using PASW Statistics, version 18.0 (SPSS Inc., Chicago, IL, USA).

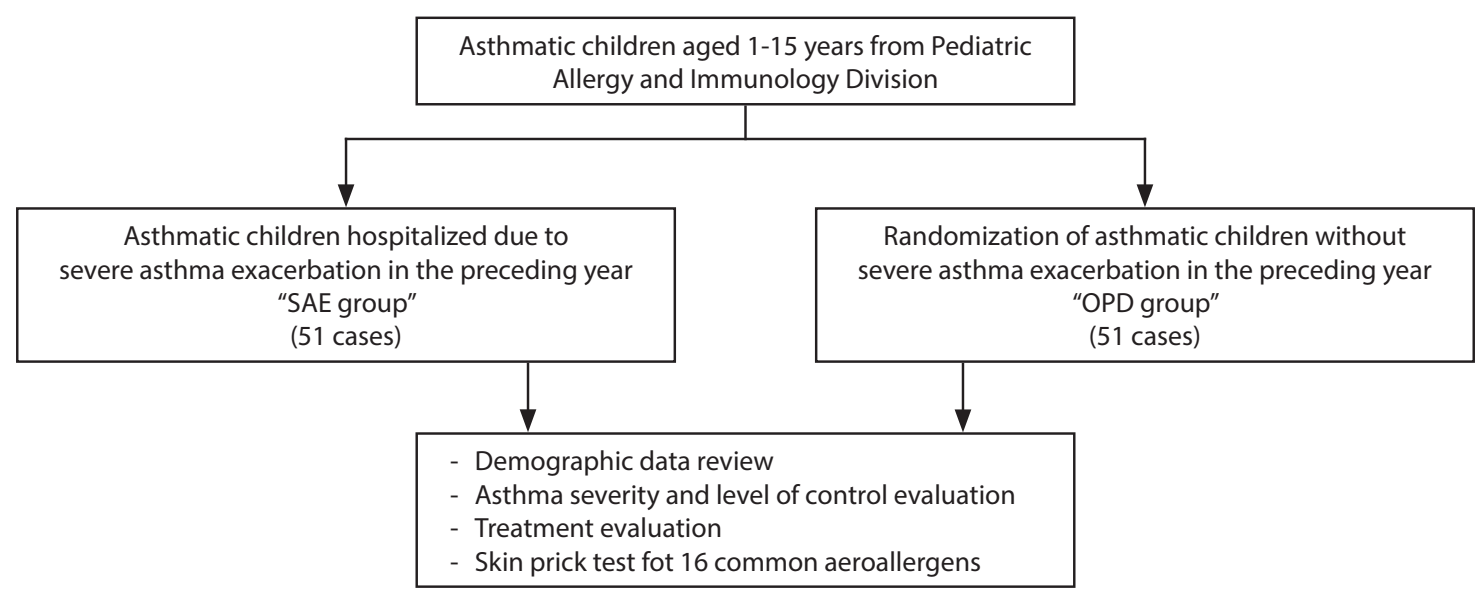

Figure 1. Flow of the participants in the study 


\section{Results}

This study enrolled 102 patients, with 51 in the SAE group and 51 in the OPD group. There were 68 males $(66.7 \%)$. The mean ages of the SAE and OPD groups were significantly different $(6.8 \pm 3.3$ years for the SAE group vs. $8.7 \pm 3.2$ years for the OPD group, $p=0.005$; Table 1). Nine patients in the SAE group were admitted to an intensive care unit for continuous nebulization due to unresponsiveness to conventional asthma therapy.
The possible factors for SAE requiring hospitalization in the previous year compared to those of the OPD group are shown in Tables 1 and 2. The proportion of patients with allergic rhinitis was significantly higher for the OPD group $(100 \%$ vs $86.3 \%, p=0.013$ ). However, a paternal history of atopy was significantly higher for the SAE group $(35.3 \%$ vs $15.7 \%, p=$ 0.023). The proportion of patients with partly controlled or uncontrolled asthma was also higher for the SAE group than

Table 1. Demographic data of the severe asthma exacerbation (SAE) and outpatient (OPD) groups

\begin{tabular}{lccc}
\multicolumn{1}{c}{ Data } & $\begin{array}{c}\text { SAE } \\
(\mathbf{N}=51)\end{array}$ & $\begin{array}{c}\text { OPD } \\
(\mathbf{N}=51)\end{array}$ & p-value \\
\hline Current age; years (mean \pm SD) & $6.8 \pm 3.3$ & $8.7 \pm 3.2$ & 0.005 \\
\hline Age of onset; years (mean \pm SD) & $3.5 \pm 2.4$ & $3.2 \pm 2.5$ & 0.512 \\
\hline Sex; male & $33(64.7 \%)$ & $35(68.6 \%)$ & 0.834 \\
\hline Obesity & $13(25.5 \%)$ & $14(27.5 \%)$ & 1.000 \\
\hline Allergic rhinitis & $44(86.3 \%)$ & $51(100 \%)$ & 0.013 \\
\hline Atopic dermatitis & $7(13.7 \%)$ & $9(17.6 \%)$ & 0.786 \\
\hline Food allergy & $4(7.8 \%)$ & $8(15.7 \%)$ & 0.357 \\
\hline Sinusitis & $13(25.5 \%)$ & $8(15.7 \%)$ & 0.221 \\
\hline Paternal history of atopy & $18(35.3 \%)$ & $8(15.7 \%)$ & 0.023 \\
\hline Maternal history of atopy & $12(23.5 \%)$ & $21(41.2 \%)$ & 0.057 \\
\hline Passive smoker & $23(44.2 \%)$ & $15(30 \%)$ & 0.137 \\
\hline Pet in house & $20(38.5 \%)$ & $20(40 \%)$ & 0.874 \\
\hline Damp house & $22(42.3 \%)$ & $22(44 \%)$ & 0.863 \\
\hline
\end{tabular}

Table 2. Severity of asthma, level of control, and the treatment of the severe asthma exacerbation (SAE) and outpatient (OPD) groups

\begin{tabular}{|lccc}
\hline \multicolumn{1}{|c}{ Data } & $\begin{array}{c}\text { SAE } \\
(\mathbf{N}=\mathbf{5 1})\end{array}$ & $\begin{array}{c}\text { OPD } \\
(\mathbf{N}=51)\end{array}$ & p-value \\
\hline Severity of asthma & & & 0.054 \\
\hline Mild asthma & $17(33.3 \%)$ & $29(56.9 \%)$ & \\
\hline Moderate asthma & $30(58.8 \%)$ & $19(37.3 \%)$ & \\
\hline Severe asthma & $4(7.8 \%)$ & $3(5.9 \%)$ & 0.015 \\
\hline Level of control & & & \\
\hline Controlled & $30(58.8 \%)$ & $42(82.4 \%)$ & \\
\hline Partly controlled & $17(33.3 \%)$ & $9(17.6 \%)$ & \\
\hline Uncontrolled & $4(7.8 \%)$ & $0(0 \%)$ & 0.243 \\
\hline Medication & & & 0.490 \\
\hline ICS & $37(72.5 \%)$ & $40(78.4 \%)$ & 0.586 \\
\hline ICS + LABA & $14(27.5 \%)$ & $10(19.6 \%)$ & \\
\hline LTRA & $9(17.6 \%)$ & $7(13.7 \%)$ & $0(0 \%)$ \\
\hline Doxofylline (Puroxan) & $3(5.9 \%)$ & & \\
\hline
\end{tabular}


Table 2. (Continued)

\begin{tabular}{|c|c|c|c|}
\hline Data & $\begin{array}{c}\text { SAE } \\
(\mathbf{N}=5 \mathbf{1})\end{array}$ & $\begin{array}{c}\text { OPD } \\
(\mathbf{N}=51)\end{array}$ & $p$-value \\
\hline ED visit in the preceding year & & & $<0.001$ \\
\hline 0 times/year & $0(0 \%)$ & $19(37.3 \%)$ & \\
\hline $1-3$ times/year & $33(64.7)$ & $27(52.9 \%)$ & \\
\hline$>3$ times/year & $18(35.3 \%)$ & $5(9.8 \%)$ & \\
\hline Systemic corticosteroid $\geq 1$ time/year & $51(100 \%)$ & $16(31.4 \%)$ & $<0.001$ \\
\hline Poor adherence & $17(33.3 \%)$ & $10(19.6 \%)$ & 0.116 \\
\hline Incorrect device method & $15(29.4 \%)$ & $7(13.7 \%)$ & 0.054 \\
\hline ICU admission for asthma* & $9(17.6 \%)$ & $4(7.8 \%)$ & 0.138 \\
\hline Hospitalization for asthma ${ }^{*}$ & $51(100 \%)$ & $34(66.7 \%)$ & $<0.001$ \\
\hline
\end{tabular}

* prior to enrolling in this study

the OPD group $(41.2 \%$ vs $17.6 \%, p=0.009)$. Similarly, both the incidence of an ED visit and a history of systemic corticosteroid administration in the preceding year were significantly higher for the SAE group (100\% vs $62.7 \%, p<0.001$; and $100 \%$ vs $31.4 \%, p<0.001$, respectively). Moreover, the incidence of hospitalization for asthma prior to enrollment in this study was significantly higher for the SAE group (100\% vs $66.7 \%, p<0.001)$. On the other hand, the two groups were not significantly different prior to study recruitment in terms of age at onset of asthma, obesity, atopic dermatitis, food allergy, sinusitis, maternal history of atopy, passive smoker, pet, damp house, low parental income (<20,000 Baht/month), severity of asthma, asthma medication, poor adherence, incorrect device method, and history of intensive care unit admission.
A nasopharyngeal wash was performed for common respiratory viruses (influenza A virus, influenza B virus, parainfluenza virus, adenovirus, and respiratory syncytial virus) during asthma exacerbation in 20 patients of the SAE group. Four patients had positive nasopharyngeal wash results: two for respiratory syncytial virus, one for adenovirus, and one for parainfluenza virus.

The 4 most common aeroallergens sensitized in the asthmatic patients were house dust mite (D. pteronyssinus and $D$. farinae; 74.5\%), cockroach (American and German cockroach; $43.1 \%)$, Alternaria $(33.3 \%)$, and cat $(24.5 \%)$. There were no statistical differences in the types of allergen sensitization evidenced by the SAE and OPD groups (Figure 2). Of the positive aeroallergen SPTs, poly-sensitization and mono-sensitization were found in $79.4 \%$ and $20.6 \%$ of cases, respectively.

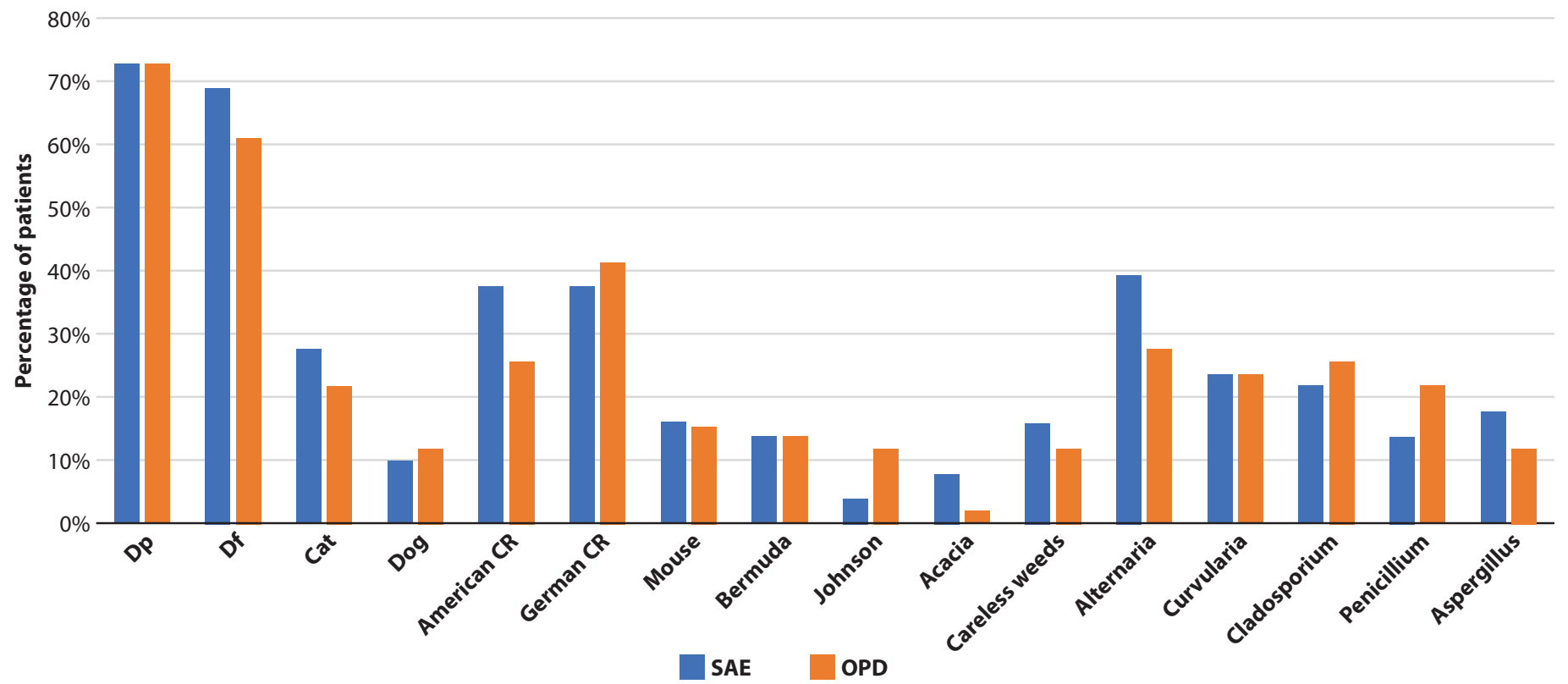

Figure 2. Allergen sensitization in the severe asthma exacerbation (SAE) and outpatient (OPD) groups. Dp, Dermatophagoides ptronyssinus; Df, Dermatophagoides farinae; CR, cockroach 
Table 3. Multivariate logistic regression analysis of factors associated with severe asthma exacerbation

\begin{tabular}{|lccc}
\hline \multicolumn{1}{|c}{ Factor } & $\begin{array}{c}\text { Adjusted } \\
\text { odds ratio }\end{array}$ & 95\% CI & p-value \\
\hline Alternaria sensitization & 3.00 & $1.09-8.3$ & 0.033 \\
\hline Age $<$ 6 years & 3.28 & $1.17-9.18$ & 0.024 \\
\hline Paternal history of atopy & 2.94 & $1.05-8.25$ & 0.040 \\
\hline Partly-controlled/uncontrolled asthma & 2.55 & $0.94-6.89$ & 0.065 \\
\hline
\end{tabular}

As to the mono-sensitized cases, house dust mite was the most common allergen (90.5\%), followed by American cockroach (9.5\%). Non-atopic asthma (negative SPT) was found in $19.6 \%$ of the patients in each group.

The multivariate logistic regression analysis of the factors associated with SAE that required hospitalization (Table 3) showed that Alternaria sensitization, an age younger than 6 years, and a paternal history of atopy were risk factors for SAE. The adjusted odds ratio (AOR) of Alternaria sensitization was 3.00 , with a $95 \%$ CI $1.09-8.3, p=0.033$. The AOR of an age younger than 6 years was 3.28 (95\% CI 1.17-9.18, $p=0.024)$, while that of a paternal history of atopy was 2.94 (95\% CI 1.05-8.25, $p=0.040)$.

\section{Discussion}

Alternaria alternata is one of the most common saprophytes worldwide and has been clinically associated with asthma. The prevalence of sensitization to Alternaria has proven difficult to estimate. The Global Asthma and Allergy European network (GA(2)LEN) study of 3,034 subjects with suspected inhalant allergy showed an approximately 9\% prevalence of Alternaria sensitization. ${ }^{14}$ In a study of more than 17,000 US citizens, positive skin test responses to Alternaria occurred in $3.6 \%$ of the population, and up to $70 \%$ of patients with fungal allergy had skin test reactivity to Alternaria. ${ }^{15}$ In another study of US inner cities, $38.3 \%$ of 12,086 asthmatic children had positive skin test results to Alternaria species. ${ }^{16}$ Sensitization to Alternaria species has been more frequently associated with persistent and severe asthma in both children and adults. ${ }^{17,18}$ Children with Alternaria sensitivity have also been shown to have heightened airway responsiveness to metacholine. ${ }^{19}$

In this case-control study, we found that patients who had been admitted because of SAE were significantly more sensitized to Alternaria than those in the OPD group. Our finding of sensitization to fungal allergens, especially Alternaria, is consistent with previous reports. Beck et al. found that children aged 4-16 years who had been hospitalized for an asthma exacerbation had an Alternaria sensitization of $58.8 \%{ }^{6}$ In addition, Black et al. reported that $54 \%$ of patients aged 18-50 years who had been admitted to an ICU with an asthma attack had a positive skin test to one or more fungal allergens, compared with $30 \%$ for patients who had not been admitted to the ICU $(p=0.005) .{ }^{9}$ The same study showed no differences in the degree of positive skin test results to grasses, cat dander, or house dust mites for cases with and without ICU admission. ${ }^{9}$
Our study showed that asthmatic children who had a paternal history of atopy were more likely (2.94 times) to have SAE. The result is similar to a birth cohort study of 476 families in the USA, in which it was found that the father's disease history, particularly his asthma history, was more strongly related to the pediatric outcomes than the mother's history. ${ }^{20}$ Previous studies have also found that persistent asthma in fathers conferred a higher risk of atopy in their children ${ }^{20}$ and that a paternal history of asthma was strongly associated with airway hyperresponsiveness in offspring. ${ }^{21}$ However, in a contrasting meta-analysis, Lim et al. showed that both a paternal and maternal history of asthma increased the offspring risk of asthma, with the effect of maternal asthma having a greater extent than paternal disease. ${ }^{22}$

In the present study, the mean age of the participants in the SAE group was significantly lower than the corresponding figure for the OPD group. This result contrasts with the findings of a study by Lyell et al., which found that asthmatic patients in an ICU group were older than those in a nonICU group (mean age \pm SD: $6.7 \pm 5.3$ vs. $4.4 \pm 3.5$ years, $p=$ $0.01) .{ }^{23}$ In addition, our study did not demonstrate a correlation between SAE and other factors that had been found by earlier studies to be associated with SAE, namely, passive smoking, ${ }^{24,25}$ viral infection, ${ }^{26}$ obesity, ${ }^{27}$ low parental incomes, ${ }^{28}$ and poor adherence. ${ }^{29}$

Our study may shed some light on the risk factors for severe asthma exacerbation in the Southeast Asian population, which could be used to advise patients and prioritize care for pediatric asthmatic patients. The limitations of this study were that the nasopharyngeal wash to detect respiratory viruses was performed in some SAE patients only, and the wash did not include rhinovirus, which commonly triggers asthma exacerbation. ${ }^{10,26}$ Other limitations were that there was a small number of participants, and that no evaluation was made of air pollution, which has been reported to affect SAE. ${ }^{30}$

\section{Conclusion}

Alternaria sensitization, an age younger than 6 years, and a paternal history of atopy were associated with SAE in Thai asthmatic children.

\section{Acknowledgments}

The authors thank Julaporn Pooliam for the statistical analysis, and all of the participants and technicians involved in the study. 


\section{Conflicts of interest}

The authors declare that there are no conflicts of interest related to this study.

\section{Funding sources}

This study was supported by a Research Development Fund and a Chalermprakait Grant from the Faculty of Medicine at Siriraj Hospital (Mahidol University, Thailand) and by an NSTDA Chair Professor Grant (P-1450624), funded by the Crown Property Bureau.

\section{References}

1. Trakultivakorn M, Sangsupawanich P, Vichyanond P. Time trends of the prevalence of asthma, rhinitis and eczema in Thai children-ISAAC (International Study of Asthma and Allergies in Childhood) phase three. J Asthma. 2007;44:609-11.

2. Chinratanapisit S, Suratannon N, Pacharn P, Sritipsukho P, Vichyanond P. Prevalence and severity of asthma, rhinoconjunctivitis and eczema in children from the Bangkok area: The Global Asthma Network (GAN) Phase I. Asian Pac J Allergy Immunol. 2019;37(4):226-31.

3. Zahran HS, Bailey CM, Damon SA, Garbe PL, Breysse PN. Vital signs: Asthma in children-United States, 2001-2016. MMWR Morb Mortal Wkly Rep. 2018;67:149-55.

4. Wong GW, Kwon N, Hong JG, Hsu JY, Gunasekera KD. Pediatric asthma control in Asia: phase 2 of the Asthma Insights and Reality in Asia-Pacific (AIRIAP 2) survey. Allergy. 2013;68:524-30.

5. Reddel HK, Taylor DR, Bateman ED, Boulet LP, Boushey HA, Busse WW, et al. An official American Thoracic Society/European Respiratory Society statement: asthma control and exacerbations: standardizing endpoints for clinical asthma trials and clinical practice. Am J Respir Crit Care Med. 2009;180:59-99.

6. Sala KA, Carroll CL, Tang YS, Aglio T, Dressler AM, Schramm CM. Factors associated with the development of severe asthma exacerbations in children. J Asthma. 2011;48:558-64.

7. Beck AF, Huang B, Kercsmar CM, Guilbert TW, McLinden DJ, Lieri MB, et al. Allergen sensitization profiles in a population-based cohort of children hospitalized for asthma. Ann Am Thorac Soc. 2015;12:376-84.

8. Visitsunthorn N, Chaimongkol W, Visitsunthorn K, Pacharn $\mathrm{P}$, Jirapongsananuruk O. Great flood and aeroallergen sensitization in children with asthma and/or allergic rhinitis. Asian Pac J Allergy Immunol. 2018;36(2):69-76.

9. O’Hollaren MT, Yunginger JW, Offord KP, Somers MJ, O'Connell EJ, Ballard DJ, et al. Exposure to an aeroallergen as a possible precipitating factor in respiratory arrest in young patients with asthma. N Engl J Med. 1991;324:35963.

10. Black PN, Udy AA, Brodie SM. Sensitivity to fungal allergens is a risk factor for lifethreatening asthma. Allergy. 2000;55:5014.

11. Fu LS, Tsai MC. Asthma exacerbation in children: a practical review. Pediatr Neonatol. 2014;55:83-91.

12. Turner S. Predicting and reducing risk of exacerbations in children with asthma in the primary care setting: current perspectives. Pragmat Obs Res. 2016;7:33-9.

13. Puranik S, Forno E, Bush A, Celedón JC. Predicting severe asthma exacerbations in children. Am J Respir Crit Care Med. 2017;195:854-9.
14. O'Driscoll BR, Hopkinson LC, Denning DW. Mold sensitization is common amongst patients with severe asthma requiring multiple hospital admissions. BMC Pulm Med. 2005;5:4.

15. Gabriel MF, Postigo I, Tomaz CT, Martinez J. Alternaria alternata allergens: markers of exposure, phylogeny and risk of fungi-induced respiratory allergy. Environ Int. 2016;89-90:71-80.

16. Gergen PJ, Turkeltaub PC, Kovar MG. The prevalence of allergic skin test reactivity to eight common aeroallergens in the US population: results from the second National Health and Nutrition Examination Survey. J Allergy Clin Immunol. 1987;80:669-79.

17. Eggleston PA, Rosenstreich D, Lynn H, Gergen P, Baker D, Kattan M, et al. Relationship of indoor allergen exposure to skin test sensitivity in inner city children with asthma. J Allergy Clin Immunol. 1998;102:563-70.

18. Bush RK, Prochnau JJ. Alternaria-induced asthma. J Allergy Clin Immunol. 2004; 113:227-34.

19. Zureik M, Neukirch C, Leynaert B, Liard R, Bousquet J, Neukirch F European Community Respiratory Health Survey Sensitisation to airborne moulds and severity of asthma: cross sectional study from European Community respiratory health survey. BMJ. 2002;325:411-4.

20. Nelson HS, Szefler SJ, Jacobs J, Huss K, Shapiro G, Sternberg AL. The relationships among environmental allergen sensitization, allergen exposure, pulmonary function, and bronchial hyperresponsiveness in the Childhood Asthma Management Program. J Allergy Clin Immunol. 1999;104:775-85

21. Alford SH, Zoratti E, Peterson E, Maliarik M, Ownby DR, Johnson CC. Parental history of atopic disease: disease pattern and risk of pediatric atopy in offspring. J Allergy Clin Immunol. 2004;114:1046-50.

22. Raby BA, Van Steen K, Celedon JC, Litonjua AA, Lange C, Weiss ST, et al. Paternal history of asthma and airway responsiveness in children with asthma. Am J Respir Crit Care Med. 2005;172:552-8.

23. Lim RH, Kobzik L, Dahl M. Risk for asthma in offspring of asthmatic mothers versus fathers: a meta-analysis. PLoS One. 2010;5:e10134.

24. Lyell PJ, Villanueva E, Burton D, Freezer NJ, Bardin PG. Risk factors for intensive care in children with acute asthma. Respirology. 2005;10:436-41.

25. van den Bosch GE, Merkus PJFM, Buysse CMP, Boehmer AL, Vaessen -Verberne AA, van Veen LN, et al. Risk factors for pediatric intensive care admission in children with acute asthma. Respir Care. 2012;57:1391-7.

26. McCarville M, Sohn MW, Oh E, Weiss K, Gupta R. Environmental tobacco smoke and asthma exacerbations and severity: the difference between measured and reported exposure. Arch Dis Child. 2013;98:510-4.

27. Murray CS, Poletti G, Kebadze T, Morris J, Woodcock A, Johnston SL, et al. A study of modifiable risk factors for asthma exacerbations: virus infection and allergen exposure increase the risk of asthma hospital admissions in children. Thorax. 2006;61:376-82.

28. Carroll CL, Uygungil B, Zucker AR, Schramm CM. Identifying an at-risk population of children with recurrent near-fatal asthma exacerbations. J Asthma. 2010;47:460-4.

29. Gold LS, Smith N, Allen-Ramey FC, Nathan RA, Sullivan SD. Associations of patient outcomes with level of asthma control. Ann Allergy Asthma Immunol. 2012;109:260-5.

30. Engelkes M, Janssens HM, de Jongste JC, Sturkenboom MC, Verhamme KM. Medication adherence and the risk of severe asthma exacerbations: a systematic review. Eur Respir J. 2015;45:396-407.

31. Orellano P, Quaranta N, Reynoso J, Balbi B, Vasquez J. Effect of outdoor air pollution on asthma exacerbations in children and adults: systematic review and multilevel meta-analysis. PLoS One. 2017;12:e0174050. 